\title{
El aprendizaje del inglés a través de la creación colaborativa de relatos digitales del ámbito turístico ${ }^{1}$
}

Beatriz Cerezo Merchán ${ }^{a}$ y Ana Sevilla Pavón ${ }^{b}$

anniversitat de València, beatriz.cerezo@uv.es y ${ }^{b}$ Universitat de València, ana.m.sevilla@uv.es

\begin{abstract}
This paper presents an educational innovation project focused on the use of digital storytelling in English for Specific Purposes teaching, specifically in English language courses for Tourism. With a socio-constructive approach and a focus on active learning, in this project students in the second, third and fourth year of the Degree in Tourism and the Double Degree in Tourism and Business Administration from the University of Valencia were invited to create tourism promotional videos reflecting their professional communicative situations. This was done through a number of interrelated tasks which included introductory activities, teamwork planning, storyboarding and script preparation, video editing, oral presentations and peer evaluation. The analysis of the data collected through field notes, collaborative work plan worksheets, assessment rubrics, opinion and assessment questionnaires completed by students and teachers, and a discussion group held among teachers, yielded positive results concerning the development of this activity and methodology. It fostered students' motivation while developing their linguistic, interpersonal, teamwork, digital and problem solving competences.
\end{abstract}

Keywords: English for Specific Purposes, educational innovation, digital storytelling, collaborative work.

\section{Resumen}

Este articulo presenta un proyecto de innovación educativa centrado en el uso del relato digital en la enseñanza del inglés para fines específicos, concretamente, en asignaturas de lengua inglesa para Turismo. Con un enfoque socioconstructivista y el foco de atención en el aprendizaje activo del alumno, en este proyecto se invitó a estudiantes de segundo, tercer y cuarto curso del Grado de Turismo y el Doble Grado de Turismo y Administración de empresas de la Universitat de València a crear vídeos de promoción turística que reflejaran situaciones comunicativas de su profesión a través de una serie de tareas interrelacionadas, que incluian actividades introductorias, planificación del trabajo en equipo, elaboración de guiones gráficos y guiones, edición de vídeos, exposiciones orales y evaluación por pares. El análisis de los datos recogidos a través de diarios de campo, fichas de planificación del trabajo colaborativo, rúbricas de evaluación, cuestionarios de opinión y

\footnotetext{
1 Este artículo presenta la experiencia llevada a cabo en el proyecto de renovación de metodologías educativas "El aprendizaje del inglés para Turismo a través de la creación colaborativa de vídeos promocionales del ámbito turístico y de cinefórum" (con referencia UV-SFPIE RMD15-309948), coordinado por la Dra. Beatriz Cerezo Merchán y financiado por la Universitat de València durante el curso académico 2015/2016.
} 
valoración realizados por estudiantes y profesoras, y un grupo de discusión entre profesoras, arrojaron resultados positivos sobre el desarrollo de esta actividad y metodología, que permitieron a los estudiantes fomentar su motivación y desarrollar sus competencias lingüisticas, interpersonales, de trabajo en equipo, digitales y de resolución de problemas.

Palabras clave: inglés para fines especificos, innovación educativa, relato digital, trabajo colaborativo.

\section{Introducción}

La importancia de las Tecnologías de la información y la comunicación (TIC) es innegable en la sociedad actual, ya que esta constituye un medio fundamental para la comunicación y el desarrollo de nuestras actividades sociales y profesionales. Nuestros alumnos son nativos digitales (Prensky, 2011), y utilizan constantemente la tecnología para comunicarse e interactuar con su entorno. En la enseñanza de lenguas, en la que los métodos basados en la didáctica de la gramática han sido reemplazados por métodos más comunicativos y centrados en el aprendizaje activo del estudiante, las TIC representan herramientas extraordinarias que se adaptan a la forma de interactuar con el mundo y a las necesidades e intereses de los estudiantes y que los motivan a emplear la lengua meta para comunicarse.

El relato digital (RD) es uno de los recursos didácticos más innovadores de las últimas décadas, empleado en el marco de enfoques socioconstructivistas de enseñanza-aprendizaje. La elaboración, aparentemente sencilla, de vídeos cortos mediante los que se comparte una historia a través del uso de imágenes, vídeos, una banda sonora y un guion narrativo (Robin, 2006) suele resultar interesante y motivadora para los alumnos. Este recurso, por lo tanto, se adapta perfectamente a los nuevos enfoques educativos y de la enseñanza de lenguas, y permite a los profesores de lengua extranjera involucrar a los alumnos en el uso de la lengua meta para comunicarse, lo que constituye uno de los mayores retos en este tipo de materias (Pascual, 2013). El empleo del RD como recurso educativo fomenta, además de competencias lingüísticas y tecnológicas, la motivación de los alumnos, y ayuda a los profesores a construir entornos de aprendizaje constructivistas que faciliten la creatividad en la resolución de problemas basada en la colaboración y la comunicación entre estudiantes. Asimismo, el uso del RD puede facilitar la implementación de metodologías integradas en el desarrollo del currículum e involucrar a los alumnos en un aprendizaje de mayor esfuerzo cognitivo (Smeda, Dakich y Sharda, 2014).

Desde su desarrollo en la década de los 90 en el Center for Digital Storytelling ${ }^{2}$, el empleo del RD se ha extendido ampliamente en diferentes contextos educativos (Robin, 2008). En la Universitat de València y la Universitat Politènica de València, por ejemplo, se han llevado a cabo diversas iniciativas en los últimos años en torno al uso del RD con fines educativos, principalmente en asignaturas dedicadas al aprendizaje de lenguas y al aprendizaje de lenguas para fines específicos (Gregori Signes, 2008). Por otra parte, como afirman Alcantud, Ricart y Gregori Signes (2014), otro de los ámbitos en los que más se ha extendido el empleo del RD es la publicidad y el marketing. En la industria del turismo, en la que la publicidad y el marketing cobran una enorme importancia, el uso del RD también se ha desarrollado ampliamente. Así, por ejemplo, blogueros, publicistas y empresas especializados en viajes y destinos explotan a diario herramientas digitales y técnicas de storytelling o narración de historias en busca de nuevas vías para atraer a los viajeros.

Este artículo presenta el proyecto de innovación educativa desarrollado en torno al uso el $\mathrm{RD}$ en las asignaturas "Comunicación en lengua inglesa para Turismo II", "Comunicación en lengua inglesa para Turismo III" y "Comunicación intercultural en lengua inglesa", integradas en el segundo, tercer y cuarto curso del Grado en Turismo y el Doble Grado en Turismo y Administración de empresas de la Universitat de València. En él participaron un

\footnotetext{
${ }^{2}$ Véase: $<\underline{\text { http: } / / \text { www.storycenter.org/> }}$
} 
total de 455 estudiantes matriculados en 13 subgrupos distintos de las asignaturas anteriormente mencionadas e impartidos por 8 profesoras $^{3}$ del Departamento de Filología Inglesa y Alemana de la Universitat de València.

Este proyecto de investigación docente analizó el impacto del uso del RD en la motivación y el aprendizaje de los alumnos de estas asignaturas. En primer lugar, se creó un marco de trabajo común y coordinado para las tres asignaturas, pero adaptado a los objetivos pedagógicos y el nivel de lengua de cada una (que oscilaba entre B1.1 y B2 del Marco Común Europeo de Referencia, Consejo de Europa, 2001). Se propusieron tareas que los estudiantes realizaron colaborativamente a lo largo del primer cuatrimestre del curso 2015/2016 para terminar creando un RD sobre un producto turístico. En el estudio se utilizaron instrumentos para la recogida de datos cualitativos y cuantitativos, que posteriormente fueron analizados tanto a través de la estadística descriptiva como de forma interpretativa. La rúbrica fue el principal instrumento que facilitó datos cuantitativos, mientras que los demás instrumentos empleados, entre los que se encuentran los diarios de campo de las profesoras, las fichas de planificación del trabajo colaborativo, los cuestionarios realizados a estudiantes y a profesoras y el grupo de discusión realizado entre las profesoras, facilitaron, principalmente, datos cualitativos. El análisis exhaustivo de los datos permitió evaluar los beneficios de la aplicación del RD en el contexto educativo específico de esta investigación. A continuación, se exponen los objetivos, el desarrollo y la evaluación del proyecto.

\section{Objetivos}

Los objetivos del proyecto fueron los siguientes:

1. Adoptar, de manera coherente y coordinada, metodologías docentes más innovadoras, en línea con enfoques pedagógicos socioconstructivistas, a través del uso del RD en tres asignaturas de lengua inglesa del Grado en Turismo y el Doble Grado en Turismo y Administración de empresas.

2. Crear un marco de trabajo y un compendio de materiales para el uso del RD en clase de lengua inglesa para Turismo en distintos niveles de aprendizaje.

3. Fomentar la motivación de los estudiantes en su aprendizaje de la lengua inglesa.

4. Mejorar las competencias lingüísticas en lengua inglesa, así como el desarrollo de las habilidades interpersonales, de trabajo en equipo, digitales y de resolución de problemas de los estudiantes.

5. Conectar el proceso de aprendizaje de la lengua inglesa con situaciones comunicativas reales del sector profesional del Turismo y de la Administración de empresas.

6. Evaluar la acción docente propuesta para identificar sus puntos fuertes y débiles y mejorarla de cara a futuras ediciones del proyecto.

\section{Desarrollo de la innovación}

A continuación, se presenta el marco de trabajo empleado, integrado tanto por las fases de la investigación como por las distintas tareas que llevaron a cabo los estudiantes para plantear y desarrollar su proyecto de RD turístico. Todas las tareas estuvieron relacionadas y orientadas a la realización del proyecto, y en todo momento la profesora actuó como facilitadora o guía, ayudando a los alumnos a construir y a "andamiar" (Vygostky, 1978; Kop y Hill, 2008) su propio aprendizaje a partir de lo aprendido en tareas anteriores, así

\footnotetext{
${ }^{3}$ Junto a la Dra. Beatriz Cerezo Merchán, coautora este artículo, estas profesoras fueron: la Dra. Elvira Ma Montañés Brunet, la Dra. Beatriz Pastor García, Cristina Botella González, Ana Castaño Sánchez, Ma Asunción Cornelles Company, Carolina Jarque Ortega y Carmen Pascual Bajo. Las autoras de este artículo quisieran expresar aquí su agradecimiento a todas ellas.

(cc) BY-NC-ND 2016, Universitat Politècnica de València
}

Congreso In-Red (2016) 
como a partir de las competencias y conocimientos adquiridos en asignaturas previas, tanto de lengua inglesa como de marketing o de gestión de empresas turísticas, entre otros.

\subsection{Planificación de la intervención}

La primera etapa del proyecto fue la de planificación de la intervención, en la que las profesoras participantes en el mismo se reunieron para hablar de la idea y diseñar el plan de trabajo. Solo una de las ocho profesoras tenía experiencia previa en el trabajo con relatos digitales en el aula, por lo que, en primer lugar, esta profesora y otra compañera del departamento también con experiencia en el uso del RD realizaron un taller introductorio para el resto de profesoras sobre el RD y su potencial en el aula de inglés para fines específicos. Posteriormente, diseñaron conjuntamente un plan de trabajo en el que se incluían los objetivos y resultados previstos del proyecto, los estudiantes con los que aplicarlo, la temática de los relatos, y las fases del proyecto y su temporalización.

En un segundo encuentro, las profesoras determinaron las tareas a realizar, el tiempo o las sesiones dedicadas a cada tarea y la temporalización; los recursos humanos, logísticos, técnicos y económicos necesarios; y la responsable o corresponsable de cada acción. Posteriormente, se creó una carpeta en GoogleDrive para facilitar el almacenamiento e intercambio de materiales del proyecto entre las profesoras ${ }^{4}$.

\subsection{Presentación del proyecto y familiarización con el concepto de RD}

La primera fase de trabajo con los alumnos se materializó en una sesión presencial de dos horas de duración en un aula de informática. Tras una breve explicación del proyecto de la asignatura y de la temática de los vídeos por parte de la profesora, los estudiantes empezaron a trabajar individualmente en una serie de tareas preparatorias, incluidas en la página web de Moodle de la asignatura en forma de WebQuest (Dodge, 1996), para familiarizarse con el concepto de RD y con su estructura. Entre estas tareas, que se fueron corrigiendo una a una en clase, se encontraba la elaboración de una definición personal de RD a partir del visionado de vídeos en Internet; la lectura de fragmentos de páginas web para identificar y resumir los principios básicos del RD; el visionado y el análisis de relatos digitales de distinto tipo y temática para identificar la temática, la estructura y los recursos audiovisuales utilizados; y la búsqueda y la valoración de vídeos de Internet relacionados con el sector de los viajes, el turismo y la administración de empresas y realizados con técnicas de narración de historias.

Por último, en la sesión se expusieron las distintas fases y tareas del proyecto, así como fechas de entrega y exposición y, a continuación, se pidió a los alumnos que formaran grupos de 4 y que empezaran a plantearse posibles temáticas para su RD. Los alumnos pudieron elegir libremente a sus compañeros, con la excepción de aquellas clases en que había estudiantes de intercambio del programa Erasmus, en las que se puso como condición que estos estuvieran repartidos en distintos equipos para promover un entorno multicultural de trabajo. La temática fue diferente para cada una de las tres asignaturas, en función de los contenidos de estas y de su relación transversal con otras asignaturas de cada curso académico. De esta forma, en segundo curso se pidió a los alumnos que hicieran un vídeo sobre turismo urbano, describiendo un destino o un tipo de turismo; en tercer curso los alumnos tenían que crear una empresa innovadora ficticia; y en cuarto curso el tema giró en torno al turismo sostenible. A continuación, se muestran las indicaciones que se les dieron a los alumnos sobre la temática de sus vídeos:

Tabla 1. Temática de los RD realizados

\footnotetext{
${ }^{4}$ Una vez finalizado el proyecto, una selección de los materiales creados durante este se almacenaron en RODERIC, el repositorio institucional de objetos de aprendizaje de la UV. Véase: http://roderic.uv.es/.
} 


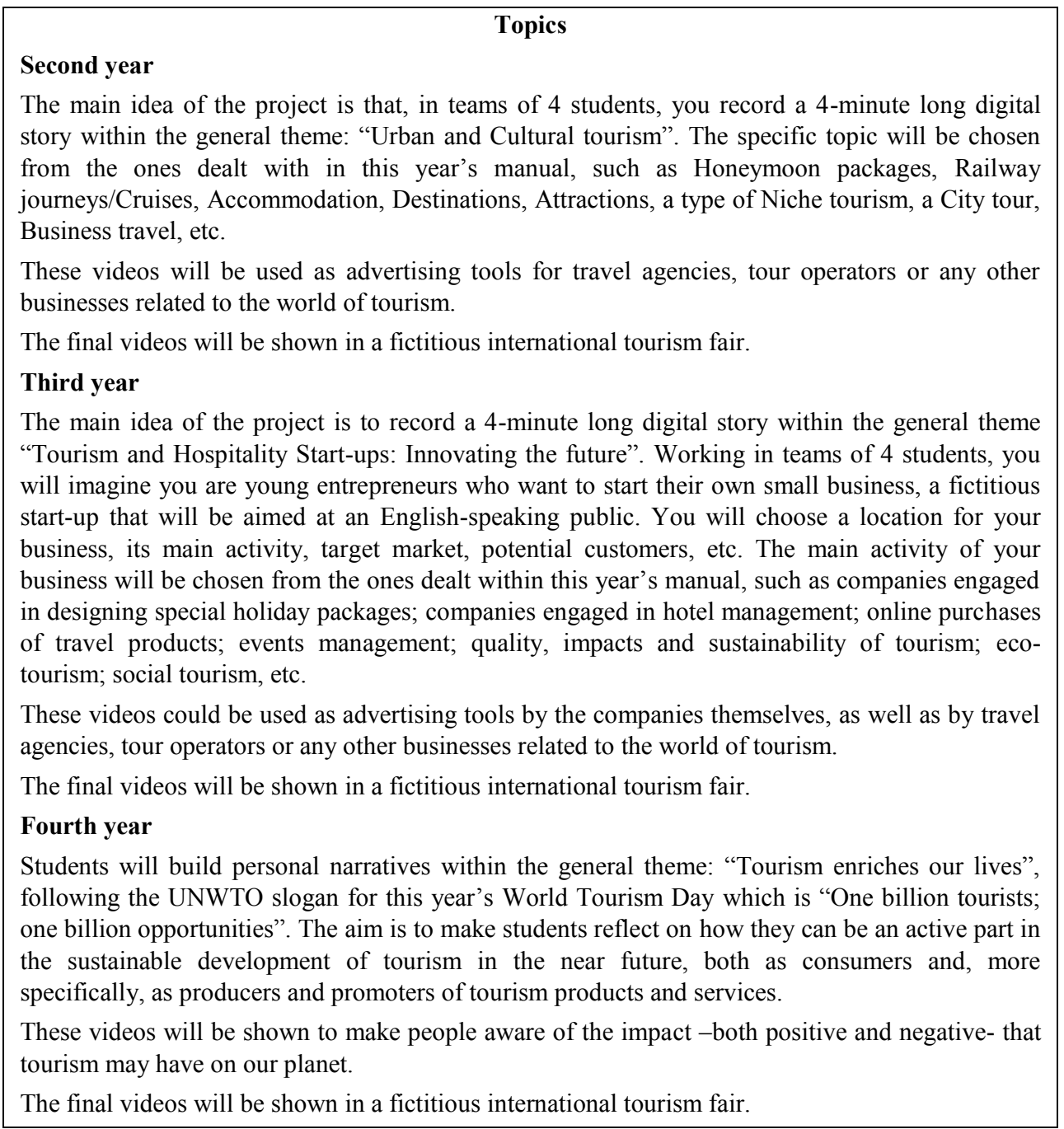

\subsection{Planificación del relato y establecimiento de compromisos}

La siguiente sesión de trabajo con los alumnos se desarrolló durante aproximadamente una hora en un aula sin ordenadores. Durante esta, los estudiantes trabajaron por equipos e hicieron una lluvia de ideas para decidir la temática y la estructura de su relato. Una vez decidida la temática y la estructura, y con el visto bueno de la profesora, los alumnos debían reflexionar sobre los recursos audiovisuales y los programas informáticos que usarían para montar su vídeo. Los estudiantes tuvieron total libertad para elegir estos programas, en función de su experiencia previa con estos y sus competencias tecnológicas. Así, muchos conocían y decidieron utilizar programas de edición de vídeo como Movie Maker, Imovie, Pinnacle o Filmora, en combinación o no con programas de edición de audio como Audacity o Iphone Dictaphone, y otros se inclinaron por programas de diapositivas, como Power Point o Prezi, que permiten combinar imágenes y fotos con sonido o con vídeos. Asimismo, algunos conocían y querían utilizar el programa PowToon, que permite diseñar presentaciones desde la nube y que funciona de manera similar a Power Point o Prezi, permitiendo la inserción de imágenes, sonido y vídeo y dándole al conjunto una apariencia similar a la de un cómic. El funcionamiento de estos programas no fue abordado en clase, pero se ofrecieron enlaces en los que los alumnos podían consultar tutoriales sobre su uso.

2016, Universitat Politècnica de València

Congreso In-Red (2016) 
Tras haber elegido la temática, la estructura, el tipo de recursos y los programas a utilizar, se pidió a cada equipo que comenzara a rellenar los huecos de la siguiente ficha, que les ayudaría a establecer compromisos y a organizar el trabajo hasta el final del proyecto y que deberían entregar completa al final del cuatrimestre:

Tabla 2. Ficha de planificación del trabajo colaborativo

\begin{tabular}{|c|c|c|c|c|}
\hline TASKS AND MEETINGS & $M E M B E R / S$ & $D A T E$ & COMPLETION TIME & $\begin{array}{l}\text { COMMENTS, PROBLEMS AND } \\
\text { SOLUTIONS TO PROBLEMS } \\
\end{array}$ \\
\hline \multicolumn{5}{|l|}{ Coordination meeting } \\
\hline & & & & \\
\hline \multicolumn{5}{|l|}{ Writing the script collaboratively } \\
\hline & & & & \\
\hline \multicolumn{5}{|l|}{$\begin{array}{l}\text { Submission of the first draft of the } \\
\text { script }\end{array}$} \\
\hline & & & & \\
\hline \multicolumn{5}{|l|}{$\begin{array}{l}\text { Submission of the subsequent } \\
\text { (corrected) versions of the script }\end{array}$} \\
\hline \multicolumn{5}{|l|}{$\begin{array}{l}\text { Video editing and recording of } \\
\text { voices }\end{array}$} \\
\hline \multicolumn{5}{|l|}{ Uploading the video to Youtube } \\
\hline \multicolumn{5}{|l|}{$\begin{array}{l}\text { Uploading the video link to } \\
\text { Moodle }\end{array}$} \\
\hline \multicolumn{5}{|l|}{$\begin{array}{l}\text { Final meeting: filling in this } \\
\text { document and preparation of class } \\
\text { oral presentation }\end{array}$} \\
\hline & & & $\begin{array}{l}\text { TOTAL } \\
\text { COMPLETION } \\
\text { TIME: }\end{array}$ & \\
\hline
\end{tabular}

\subsection{Elaboración del guion gráfico y redacción del guion}

La tercera sesión de trabajo en clase sobre el proyecto se realizó en un aula de informática durante dos horas. Durante esta, en primer lugar, se explicó qué era un guion gráfico o storyboard y por qué resultaba útil para establecer una línea argumental entre cada una de las escenas y la narración de su vídeo. Tras ver juntos algunos ejemplos de guiones gráficos, los alumnos se dispusieron a elaborar los suyos propios. A continuación, se mostraron también algunos ejemplos de guiones de relatos y se indicó que el guion debía: 1) ser el elemento más importante de su relato, 2) ser original y atractivo, 3) contener una redacción cuidada desde el punto de vista ortográfico y gramatical, 4) contener entre 400 y 500 palabras (que se corresponderían con unos 4 minutos de vídeo) y 5) redactarse colaborativamente, con la participación activa de todos los miembros del equipo. Teniendo estas premisas en mente y siguiendo diferentes estrategias de escritura colaborativa (véase Sevilla Pavón, 2015), los estudiantes empezaron a redactar sus guiones en clase, tarea que habrían de terminar autónomamente fuera del aula y entregar a la profesora a través del Sistema de Gestión del Aprendizaje Moodle. Se recomendó la utilización de GoogleDrive para la escritura colaborativa del guion, de manera cada miembro pudiera acceder siempre a un mismo documento, sobre el que se harían todas las ediciones. Asimismo, también se recomendó a los equipos el uso de GoogleDrive, Dropbox o plataformas similares para almacenar y compartir todos los materiales relativos al proyecto. 


\subsection{Corrección del guion}

La siguiente sesión de trabajo tuvo lugar durante una hora en un aula de informática. Para su corrección se siguió el mismo sistema empleado para corregir el resto de producciones escritas entregadas por los alumnos en la signatura, con el que los alumnos están familiarizados. Se usaron plantillas de autocorrección con códigos para marcar los errores de vocabulario, gramática, estilo, puntuación, etc. de las redacciones sin ofrecer las soluciones, y fueron los propios alumnos los que, con ayuda de sus compañeros, de la profesora y de las fuentes de documentación a su alcance, corrigieron sus guiones. Tras realizar las correcciones pertinentes, los estudiantes subieron la nueva versión de su guion a Moodle, versión que volvería a ser revisada por la profesora para dar su visto bueno o para proponer nuevos cambios.

\subsection{Creación de los relatos digitales}

Esta fase se realizó de manera autónoma por los estudiantes, fuera del aula, y en ella se llevaron a cabo tres tareas: la edición de los elementos audiovisuales seleccionados para crear un vídeo mediante un programa de edición de vídeo o de diapositivas; la grabación de la narración oral del guion mediante el programa mismo de edición de vídeo o un programa específico de audio instalado en el teléfono móvil o el ordenador; y, por último, la incrustación y sincronización del audio en el vídeo con el programa de edición de vídeo o diapositivas elegido. Para garantizar una correcta pronunciación de todas las palabras, se recomendó a los estudiantes que practicaran en equipo, que consultaran diccionarios en línea y que acudieran a pedir ayuda en el horario de atención de sus profesoras si lo consideraban necesario.

Una vez que los estudiantes tuvieron sus vídeos listos y en el formato adecuado, se les pidió que los subieran a Youtube en formato oculto y que, posteriormente, enviaran a la profesora el enlace al vídeo a través de la página de Moodle de la asignatura. Esta entrega, junto a la de la ficha de planificación del trabajo colaborativo rellenada, constituyeron las dos últimas tareas que los alumnos enviaron a la profesora para su revisión y evaluación.

\subsection{Presentación de los vídeos y evaluación por pares}

Basándose en las anotaciones recogidas en la ficha de planificación y en su propia experiencia, los alumnos prepararon una exposición oral breve, que sirvió como presentación de su proyecto durante la sesión final del proyecto. Cada equipo realizó su exposición y presentó su vídeo, y todos los equipos evaluaron el trabajo de los demás equipos empleando la siguiente rúbrica:

Tabla 3. Rúbrica de evaluación de los RD

\begin{tabular}{|c|c|c|c|}
\hline \multicolumn{4}{|l|}{ TOPIC } \\
\hline $\begin{array}{l}\text { Interest } \\
3 \text { very interesting } \\
2 \mathrm{OK} \\
1 \text { boring }\end{array}$ & $\begin{array}{l}\text { Originality } \\
3 \text { very original } \\
2 \mathrm{OK} \\
1 \text { not original }\end{array}$ & $\begin{array}{l}\text { Relevance } \\
3 \text { very relevant } \\
2 \mathrm{OK} \\
1 \text { not relevant }\end{array}$ & $\begin{array}{l}\text { Structure/pace } \\
3 \text { excellent } \\
2 \text { OK } \\
1 \text { not well organized }\end{array}$ \\
\hline \multicolumn{4}{|l|}{ RESOURCES } \\
\hline $\begin{array}{l}\text { Variety } \\
3 \text { lots of variety } \\
2 \text { varied } \\
1 \text { all the same }\end{array}$ & $\begin{array}{l}\text { Correct use } \\
3 \text { very well used } \\
2 \mathrm{OK} \\
1 \text { not used well }\end{array}$ & $\begin{array}{l}\text { Images } \\
3 \quad \text { attractive and } \\
\text { coherent } \\
2 \mathrm{OK}\end{array}$ & $\begin{array}{l}\text { Soundtrack } \\
3 \text { very good \& correct } \\
\text { volume } \\
2 \mathrm{OK}\end{array}$ \\
\hline
\end{tabular}

2016, Universitat Politècnica de València

Congreso In-Red (2016) 


\begin{tabular}{|c|c|c|c|}
\hline & enough & $\begin{array}{l}1 \text { not attractive or } \\
\text { coherent }\end{array}$ & $\begin{array}{l}1 \text { not well used/too } \\
\text { loud }\end{array}$ \\
\hline \multicolumn{4}{|l|}{ VOICE } \\
\hline $\begin{array}{l}\text { Pacing } \\
3 \text { very good pace } \\
2 \mathrm{OK} \\
1 \text { too fast/slow }\end{array}$ & $\begin{array}{l}\begin{array}{l}\text { Language use } \\
\text { grammar }\end{array} \\
3 \text { very good vocab } \& \\
\text { structures } \\
2 \text { OK } \\
1 \text { poor vocab \& } \\
\text { structures }\end{array}$ & $\begin{array}{l}\text { Pronunciation } \\
\text { intonation } \\
3 \text { very good, } \\
2 \text { OK } \\
1 \text { poor, difficult to } \\
\text { understand }\end{array}$ & \\
\hline
\end{tabular}

Con la evaluación por pares (Sevilla Pavón, Serra Cámara y Gimeno Sanz, 2012) se persiguió hacer a los alumnos protagonistas del proyecto hasta el final, hacerles reflexionar sobre la calidad de los diferentes elementos de los relatos e involucrarlos en la evaluación. Tras rellenar la rúbrica, cada equipo votó el mejor relato digital, el relato más original, el relato con los mejores recursos audiovisuales y el relato con mejor uso del inglés. Los mejores relatos de cada uno de los grupos participantes en el proyecto participarían, unos días más tarde, en un concurso final al que todos los estudiantes de segundo, tercer y cuarto curso podían asistir si así lo deseaban.

\subsection{Concurso final}

El concurso final de relatos digitales tuvo lugar en un salón de actos de la universidad de gran aforo, al objeto de que pudiera asistir el mayor número posible de alumnos para ver los vídeos de los equipos ganadores de cada curso. Se formó un jurado compuesto por profesores expertos en el ámbito del turismo y la administración de empresas y por profesores especializados en la enseñanza de la lengua inglesa, quienes evaluaron la calidad de los RD y votaron el relato ganador y finalista de cada curso. Todos los participantes en el concurso recibieron un diploma de participación y un pequeño obsequio institucional.

\subsection{Cumplimentación del cuestionario final de opinión y valoración de la experiencia por parte de estudiantes $y$ de profesoras}

Al término del proyecto, se pidió a estudiantes y profesoras que rellenaran un cuestionario final de opinión y valoración de la experiencia cuyas preguntas se presentan a continuación.

Cuestionario para alumnos:

1. Did you like the activity?

2. Please, rate your experience from 1 to 5 ( 1 being "very negative" and 5 being "very positive")

3. What did you enjoy most?

4. What didn't you like at all?

5. Do you think it has improved your English? Why/why not?

6. What other things have you learnt from it?

7. How would you improve the experience?

8. Any other comments?

Cuestionario para profesoras:

1. Do you think that digital storytelling is a good educational tool for language learning?

2. Please, rate your experience with the use of digital storytelling in class this year from 1 to 5 (1 being "very negative" and 5 being "very positive")?

3. What was the most positive aspect of your experience? 
4. What was the most negative aspect of your experience?

5. Do you think that digital storytelling has helped students improve their language skills?

6. What other skills do you think they have developed?

7. How would you improve the project?

8. Any other comments?

\subsection{Recogida de datos, análisis de resultados y grupo de discusión}

El último paso del proyecto consistió en la recogida y análisis de datos de los tres estudios de caso aquí presentados. Posteriormente, las profesoras realizaron un grupo de discusión para reflexionar sobre la experiencia, contrastar resultados y desarrollar propuestas de mejora y líneas de acción para el futuro.

\section{Resultados}

A lo largo del proyecto se recogieron datos cuantitativos y cualitativos tanto de los estudiantes como de las profesoras participantes en la experiencia a través de: 1) diarios de campo, 2) una rúbrica de evaluación de los relatos digitales, 3) una ficha de planificación del trabajo colaborativo de los alumnos, 4) un cuestionario final de opinión y valoración de la experiencia a los estudiantes, 5) un cuestionario final de opinión y valoración de la experiencia a las profesoras, y 6) un grupo de discusión con las profesoras para analizar los resultados del proyecto.

Dada la elevada cantidad de datos recogidos, para la elaboración de este artículo se seleccionó aleatoriamente una clase o grupo de cada curso, es decir, un grupo de segundo curso (del Grado en Turismo y Administración de empresas), uno de tercero (del Grado en Turismo) y uno de cuarto (del Grado de Turismo). El total de la muestra de la población ascendió a 80 estudiantes, cuyos datos se analizaron de manera exhaustiva y se presentan a continuación. Este análisis que se presenta no es, sin embargo, comparativo, puesto que no es objetivo del proyecto comparar los resultados obtenidos en los tres niveles educativos en los que se ha trabajado. Por otra parte, aunque no podemos extrapolar los resultados de estos tres estudios de caso al conjunto de la población de todos los grupos de alumnos que participaron en el proyecto, el grupo de discusión organizado entre las profesoras para observar los resultados de los tres estudios de caso puso de manifiesto que dichos resultados y las conclusiones derivadas de estos concordaban con la experiencia de todas las profesoras que participaron en el estudio, fueran o no sus grupos parte de los seleccionados aleatoriamente para el análisis exhaustivo que se presenta en este artículo. Por ello, se puede intuir que los datos de esta muestra son representativos del conjunto de la población.

A continuación, se presentan los principales resultados y conclusiones del proyecto, vinculados directamente con los objetivos de la investigación que, como se ha señalado anteriormente, fueron analizar las percepciones y valoración sobre la experiencia de todos los implicados en el proyecto, estudiantes y profesoras, así como evaluar el impacto del uso del $\mathrm{RD}$ en la motivación de los alumnos y en su desarrollo de competencias lingüísticas, interpersonales, de trabajo en equipo, digitales y de resolución de problemas.

\subsection{Opinión y valoración de la experiencia por parte de estudiantes y profesoras}

A continuación, se presentan los resultados referentes a la opinión y valoración por parte de los estudiantes y de las profesoras de la experiencia con el uso del RD en el aula de lengua inglesa para Turismo. Los datos cualitativos y cuantitativos que aquí se exponen fueron recabados a partir de los cuestionarios realizados a estudiantes y profesoras, así como del grupo de discusión realizado entre las profesoras.

2016, Universitat Politècnica de València

Congreso In-Red (2016) 


\subsubsection{Opinión y valoración de los estudiantes}

Según los datos recabados en los cuestionarios, la valoración de la experiencia fue muy alta, situándose en 4,5 sobre 5 puntos, de lo que se desprende que la actividad agradó y motivó a la gran mayoría de los estudiantes. Esta opinión, coincide, como se verá a continuación, con aquella de las profesoras.

A excepción de los estudiantes de cuarto, que habían participado en una experiencia con relatos digitales en el primer curso del grado, se trató de una experiencia nueva para todo el alumnado. Y fue precisamente la novedad de esta metodología educativa uno de los aspectos que valoraron más positivamente sobre la actividad. Otros aspectos positivos que la mayor parte de los estudiantes señalaron en los cuestionarios fueron que la actividad les había permitido implicarse más activamente en su aprendizaje, ser más creativos y aprender inglés de una forma divertida, además de aprender a aplicar estrategias de marketing para hacer vídeos interesantes en el ámbito del turismo y la administración de empresas.

Pero fueron, sin lugar a dudas, los aspectos relativos a las relaciones interpersonales los que los estudiantes afirmaron haber disfrutado más durante la actividad. Estos señalaron lo mucho que les había gustado pasar tiempo con sus compañeros dentro y fuera de clase planeando y haciendo el vídeo, la posibilidad que les había dado de conocer mejor a sus compañeros, y lo divertido y motivador que les resultó poder mostrar su vídeo en clase y ver los vídeos de los compañeros.

Asimismo, otros aspectos que valoraron positivamente, aunque en menor frecuencia, fueron la posibilidad que les había dado la experiencia de mejorar sus habilidades de gestión de trabajo en equipo; la libertad que habían tenido para crear un vídeo muy personal, eligiendo ellos mismos el tema, estructura, estilo, etc.; la implicación de las profesoras en todo el proceso; y la idea de hacer un concurso final de vídeos.

Por otra parte, entre los aspectos que menos gustaron a los alumnos destacan las repetidas revisiones que algunos equipos tuvieron que realizar de su guion hasta conseguir el visto bueno de la profesora, los problemas técnicos que otros equipos experimentaron a la hora de editar el vídeo y el tiempo total que invirtieron en la actividad, no suficientemente reconocido en los porcentajes de evaluación de la asignatura (el proyecto de RD solo representaba el $15 \%$ de la nota final). Estos problemas se relacionan directamente con sus propuestas de mejora de la actividad, que giran en torno a la ampliación del tiempo dedicado en clase a la elaboración del guion y al uso de programas informáticos de edición de vídeo, y al aumento del porcentaje de evaluación continua correspondiente a la actividad. En futuras ediciones del uso del RD en el aula de inglés para Turismo, estos aspectos serán, sin lugar a dudas, tenidos en cuenta para la mejora de la experiencia.

\subsubsection{Opinión y valoración de las profesoras}

Las ocho profesoras que participaron en el proyecto valoraron muy positivamente el potencial pedagógico del RD, aunque nunca, con excepción de una de ellas, lo hubieran empleado en clase antes. En el grupo de discusión, varias profesoras manifestaron, además, que desde hacía tiempo deseaban probar esta metodología, ya que la consideraban diferente, innovadora, creativa y divertida, lo que vendría muy bien para incrementar el interés de ciertos grupos de estudiantes poco motivados por el uso del inglés.

En cuanto a la valoración global de las profesoras sobre la experiencia con el uso del RD, esta se sitúa en un 4,75 sobre 5. Con una puntuación media ligeramente superior a la otorgada por los estudiantes, este dato permite concluir que la actividad fue también del agrado de las profesoras.

Por otra parte, los aspectos de la experiencia valorados más positivamente fueron la innovación que suponía esta actividad, mediante la que los alumnos podían trabajar las destrezas del inglés de forma más creativa y con las TIC, etc.; el incremento de motivación 
que observaron en los alumnos con respecto a aquellos de cursos anteriores en las mismas asignaturas; la coordinación curricular establecida entre las tres asignaturas de lengua inglesa para Turismo del estudio; y la relación y el apoyo entre todas las profesoras del proyecto.

El aspecto valorado más negativamente, que se repitió en todos los cuestionarios, fue la gran carga de trabajo y el esfuerzo que el proyecto supuso para las profesoras, sobre todo en el seguimiento de la parte lingüística. En el grupo de discusión, las profesoras mencionaron que algunos grupos llegaron a mandar sus guiones hasta cuatro veces y seguían teniendo fallos o que los alumnos no daban excesiva importancia a las correcciones. Posibles soluciones a este problema serían ofrecer más apoyo o seguimiento de la primera corrección realizada por los alumnos, tanto en clase como en tutorías, y ofrecer directamente las soluciones a los errores cometidos en la segunda versión del guion, para evitar tener que hacer el seguimiento de una tercera o una cuarta versión.

Por último, las dos propuestas de mejora mencionadas por las profesoras fueron, por una parte, plantear la actividad con más tiempo, de modo que los plazos fueran más cómodos para los estudiantes y las profesoras, y por otra, contar con un asesoramiento más eficaz en la parte técnica (grabación y edición), tanto para profesoras como para alumnos. La primera propuesta se podría llevar a la práctica fácilmente en futuras ediciones del proyecto, puesto que ya se cuenta con la experiencia de este curso, un marco de trabajo y un compendio de materiales docentes listos para usar. En cuanto a la segunda, una posible solución sería realizar uno o varios talleres extracurriculares con personal técnico de la universidad que ayudase a alumnos y profesoras en el uso de programas básicos de edición de vídeo.

\subsection{Impacto del RD en el aprendizaje de los alumnos}

En este apartado se presentan los resultados relativos al impacto del uso del RD en el aprendizaje de los alumnos, fundamentalmente en su motivación y en el desarrollo de sus competencias lingüísticas, interpersonales, de trabajo en equipo, digitales y de resolución de problemas.

\subsubsection{Notas globales en el proyecto de $R D$}

En primer lugar, se exponen las calificaciones medias obtenidas en el proyecto del RD por los tres grupos estudiados. En la calificación global de cada grupo se tuvieron en cuenta, además de la calidad de los relatos, las exposiciones orales en clase, la entrega puntual de todas las tareas, y la implicación y participación de los estudiantes.

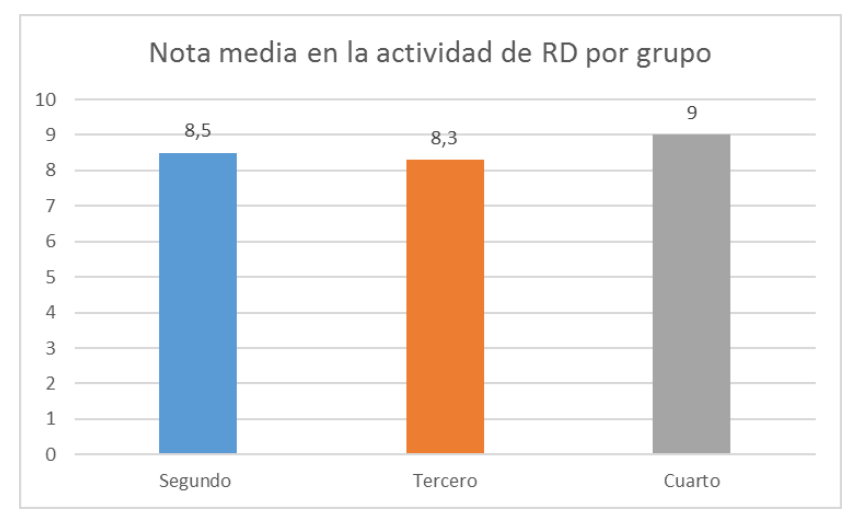

Fig. 1 Nota media en la actividad de RD por grupo

2016, Universitat Politècnica de València

Congreso In-Red (2016) 
Como se puede ver en el gráfico, las calificaciones finales de la actividad del RD son bastante elevadas (más de un 8 sobre 10) y similares en los tres casos, aun tratándose de grupos de alumnos diferentes, en distintos niveles educativos y con distintas profesoras. Estos resultados tan positivos nos llevan a pensar que este tipo de actividad y metodología tiene un impacto positivo en el aprendizaje de los alumnos. No obstante, para medir cuál ha sido realmente la influencia de la experiencia con el RD en el aprendizaje de los alumnos habría sido necesario estudiar, por ejemplo, las calificaciones de los exámenes finales escritos y orales de estos grupos con respecto al año académico anterior. Desafortunadamente, la fluctuación de alumnos y grupos debida a los cambios de matrícula en todos los cursos y a que la asignatura de cuarto es optativa hizo que esta comparación no se llegase a realizar por no resultar estadísticamente adecuada. En futuras ediciones del proyecto se recurrirá al uso de grupos de control para poder realizar comparaciones estadísticamente significativas y estudiar la influencia del uso del RD sobre los resultados de las pruebas escritas y orales finales de la asignatura.

\subsubsection{Resultados globales en los distintos elementos evaluados del $R D$}

El siguiente gráfico muestra las puntuaciones medias (del 1 al 3) obtenidas en los diferentes elementos evaluados del RD en los tres estudios de caso. Estas puntuaciones fueron otorgadas por las profesoras, quienes en su tarea tuvieron también en cuenta las adjudicadas por los alumnos durante la presentación de los vídeos. Los datos revelan que los cuatro elementos referentes al tema (interés, originalidad, relevancia y estructura) fueron los que mayor puntuación (entre 2,5 y 3) registraron en todos los grupos. Esto refleja el buen trabajo de planificación previo realizado por los equipos, sobre todo en la elección del tema y en la elaboración del guion gráfico. Los cuatro elementos relativos a los recursos audiovisuales (variedad, uso correcto, imágenes y pista sonora) recibieron, sin embargo, puntuaciones más bajas (comprendidas entre el 2 y el 2,5) en comparación con las anteriores. La pista sonora fue el elemento peor valorado, lo que probablemente se debe a que, como se expondrá en el siguiente apartado, los estudiantes encontraron uno de sus principales problemas técnicos en la sincronización del audio con el vídeo. Por último, los elementos de la lengua (ritmo, uso de la lengua y gramática y pronunciación y entonación) recibieron puntuaciones comprendidas entre el 2 y el 3. En los grupos de segundo y tercero, el ritmo fue el elemento mejor valorado de entre los lingüísticos, mientras que la pronunciación y entonación, uno de los peor valorados en todo el RD. En el grupo de cuarto se registraron, sin embargo, resultados opuestos: el uso de la lengua y la gramática, y la pronunciación y entonación sobresalieron y alcanzaron la máxima puntuación. Este hecho se debe, probablemente, a que la asignatura de cuarto curso es optativa y a que en ella suelen matricularse aquellos estudiantes más motivados y avanzados en el aprendizaje de la lengua inglesa. 


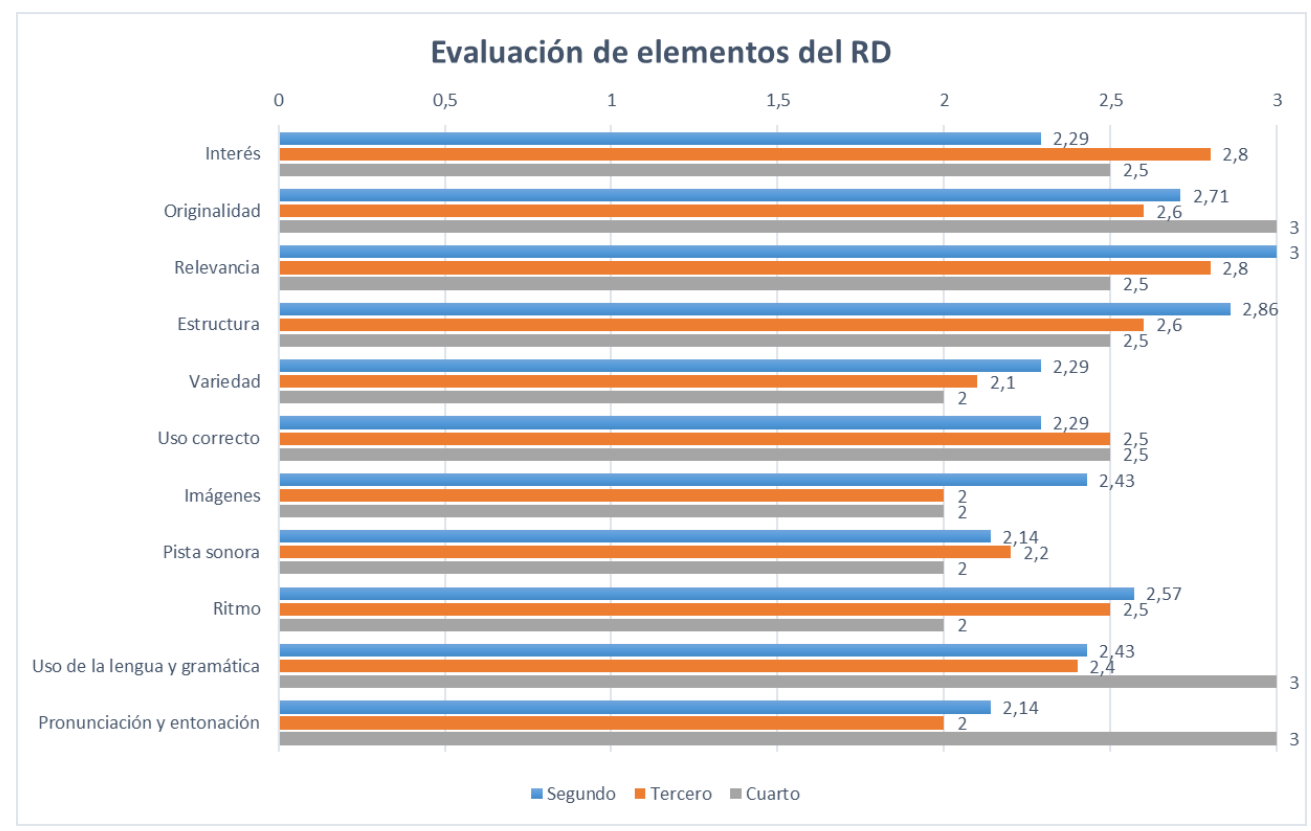

Fig. 2 Evaluación de elementos del RD

4.2.3. Percepciones sobre el impacto del RD en la motivación y el desarrollo de competencias por parte de los estudiantes

\section{Motivación}

De los datos recabados en los cuestionarios a estudiantes, quienes valoraban la actividad en un 4,5 sobre 5, y a profesoras, quienes apuntaban haber observado un incremento de motivación en los alumnos con respecto a aquellos de cursos anteriores en las mismas asignaturas, se puede desprender que este tipo de actividad y metodología ha fomentado el interés y la motivación de los alumnos. La novedad, la creatividad, el uso de las TIC y el trabajo en equipo fueron, como ya se ha visto anteriormente, algunos de los aspectos mejor valorados y que más motivaron a los alumnos durante la elaboración de su RD.

\section{Competencias lingüísticas}

En primer lugar, en cuanto a las competencias lingüísticas, cuando se preguntó a los alumnos en el cuestionario si consideraban que habían mejorado su inglés, aproximadamente la mitad de ellos contestó negativamente y la otra mitad, afirmativamente. Los que respondieron negativamente argumentaron que cada estudiante solo había narrado un minuto de audio en el vídeo o que cuando escribieron su guion se limitaron a "hacerlo fácil", sin esforzarse por buscar o utilizar nuevo vocabulario o fraseología.

Por su parte, los que respondieron afirmativamente explicaron que habían intentado plasmar en el guion el vocabulario y la gramática aprendida en la asignatura, que las correcciones del guion les habían ayudado a identificar errores recurrentes y a corregirlos, y que los continuos ensayos realizados antes de grabar el audio en el vídeo les habían servido para mejorar y para ser más conscientes de la importancia de lanzarse a hablar más y de una correcta pronunciación. Asimismo, los estudiantes más tímidos valoraron muy positivamente la

2016, Universitat Politècnica de València

Congreso In-Red (2016) 
oportunidad de perder el miedo a hablar en inglés de una forma divertida y relajada, y también la ayuda de sus compañeros con la escritura y la pronunciación.

Las profesoras fueron, en general, más positivas que los estudiantes en este aspecto, ya que todas consideraron que los estudiantes habían mejorado sus competencias lingüísticas, pese a que, obviamente, se trataba de una actividad limitada en cuanto al tiempo y el volumen de trabajo dedicados.

\section{Competencias interpersonales y de trabajo en equipo}

En segundo lugar, como veíamos anteriormente en el apartado 4.1., la interacción con los compañeros y el trabajo en equipos fueron los aspectos mejor valorados por alumnos. Las profesoras, por su parte, también valoraron muy positivamente las habilidades interpersonales y de trabajo en equipo desarrolladas por los estudiantes.

El análisis de las fichas de planificación del trabajo en equipo permitió observar que las actividades fueron realizadas de manera colaborativa y que los problemas fueron resueltos en común o con la ayuda de alguno de los compañeros, más experto en un área concreta. Investigaciones previas, como la de Miller (2009), apuntan los beneficios de este tipo de dinámicas de equipo, en las que unos alumnos aprenden gracias a la orientación o guía de un compañero experto, a la vez que este compañero se siente motivado para emplear, enseñar y desarrollar su mejor habilidad.

\section{Competencias digitales}

El desarrollo de las competencia digital fue también fundamental en este proyecto, en línea con las recomendaciones institucionales respecto a iniciativas educativas que permitan el desarrollo de habilidades "que van desde el acceso a la información hasta su transmisión en distintos soportes una vez tratada, incluyendo la utilización de tecnologías de la información y comunicación como elemento esencial para informarse, aprender y comunicarse" (Real Decreto 1631/20067, de 29 de diciembre, Ministerio de Educación, Gobierno de España, 2006: 688). Los datos recabados tanto en los cuestionarios a alumnos y profesoras como en las fichas de planificación de la actividad reflejan que los estudiantes tuvieron la oportunidad de mejorar su competencia digital a través del uso de ordenadores, móviles, cámaras y diferentes programas de edición de imágenes y de vídeo.

\section{Competencias de resolución de problemas}

Tal y como queda reflejado en los cuestionarios y en las fichas de planificación del trabajo en equipo, la actividad del RD planteó nuevas situaciones y nuevos desafíos para la mayoría de los alumnos, sobre todo en el plano lingüístico y tecnológico. Para hacer frente a estos retos, los estudiantes tuvieron que poner en marcha estrategias de resolución de problemas como, por ejemplo, en caso de no estar familiarizados de algún programa de edición de vídeo, leer o visualizar tutoriales en Internet o pedir ayuda a algún compañero (de su equipo o de otros equipos), quien actuaba como tutor tecnológico.

\section{Conclusiones}

El presente artículo muestra la experiencia llevada a cabo con el RD en tres asignaturas de lengua inglesa del Grado en Turismo y el Doble Grado en Turismo y Administración de empresas de la Universidad de Valencia. En relación con los objetivos de investigación, del estudio se derivan una serie de conclusiones, que se presentan a continuación. 
En primer lugar, se puede concluir que ha sido posible adoptar, de manera coherente y coordinada, metodologías docentes más innovadoras a través del uso del RD en las tres asignaturas, y que se ha creado un marco de trabajo y un compendio de materiales que ayudarán a futuros profesores y a alumnos a experimentar los beneficios del uso del RD en el aula de lengua inglesa para Turismo en el futuro. En este sentido, la coordinación entre las profesoras implicadas y el apoyo institucional fueron elementos clave para el éxito del proyecto.

Por otra parte, esta investigación demuestra que el uso del RD ha permitido fomentar la motivación de los estudiantes, así como el desarrollo de las competencias lingüísticas, interpersonales, de trabajo en equipo, digitales y de resolución de problemas de los estudiantes. Por ello, es posible concluir, en línea con lo descubierto por otros investigadores como Garrad (2011), que el uso del RD se ajusta a enfoques pedagógicos socioconstructivistas y que es una metodología de enseñanza adecuada con un impacto positivo en el aprendizaje de los estudiantes. Asimismo, si tenemos en cuenta la importancia creciente que existe en la actualidad del formato vídeo y del uso de estrategias de narración de historias en el sector del marketing turístico, resulta innegable el valor y el impacto que tiene este tipo de actividades en la formación de los futuros profesionales del turismo.

\section{Referencias}

AlcAntud, M., RicART, A. y GREgori Signes, C. (2014). “'Share your experience'. Digital Storytelling in English for Tourism”. Ibérica, 27, 185 -204. $<$ http://www.aelfe.org/documents/09 27 Alcantud.pdf $>$ [Consulta: 18 de marzo de 2016].

CONSEJO DE EUROPA (2011). Common European Framework of Reference For Languages: Learning, Teaching And Assessment. Cambridge: Cambridge University Press. $<$ https://www.coe.int/t/dg4/linguistic/Source/Framework_EN.pdf $>$ [Consulta: 18 de marzo de 2016].

DodGe, B. (1995). Some Thoughts About WebQuests. $<$ http://edweb.sdsu.edu/courses/edtec596/about webquests.html $>$ [Consulta: 18 de marzo de 2016].

GARRARD, D. (2011). A case study to evaluate the effectiveness of digital storytelling as a narrative writing tool. Tesis doctoral. Limerick: University of Limerick,

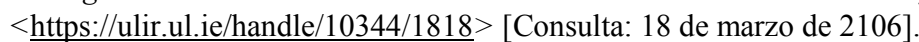

Gregori Signes, C. (2008). "Dos Proyectos para el eportfolio: El relato digital y aprende cantando" en Jornada Nacional sobre Estudios Universitarios: de los proyectos de convergencia a la realidad de los nuevos títulos. Castelló de la Plana. $<$ http://www.uv.es/gregoric/DIGITALSTORYTELLING/DIGITAL STORY INDEX.html> [Consulta: 18 de marzo de 2016].

Kop, R. y HiLl, A (2008). "Connectivism: Learning theory of the future or vestige of the past?" The International Review of Research in Open and Distance Learning, 9 (3). $<$ http://www.irrodl.org/index.php/irrodl/article/view/523/1103> [Consulta: 18 de marzo de 2016].

Miller, E. (2009). Digital Storytelling. Trabajo Final de Máster. Iowa: University of Northern Iowa. $<\underline{\text { http://www.uni.edu/icss/researchhelps/miller.pdf }>}$ [Consulta: 18 de marzo de 2016].

Ministerio de Educación, Gobierno de España. (2006). Real Decreto 1631/2006 de 29 de diciembre por el que se establecen las enseñanzas mínimas correspondientes a la Educación Secundaria Obligatoria. BOE, 5, 677-773. Disponible en: $<$ https://www.boe.es/diario boe/txt.php?id=BOEA-2007-238> [Consulta: 18 de marzo de 2016].

2016, Universitat Politècnica de València

Congreso In-Red (2016) 
Pascual, B. (2013). "Digital Storytelling in Foreign Language Teaching" en Gregori Signes, C. y Alcantud, M. (eds.) Experiencing Digital Storytelling. Valencia: JPM Ediciones.

Prensky, M. (2001). Digital natives, digital immigrants. On the Horizon, 9 (5), 1-6.

Robin, B. R. (2008). "Digital Storytelling: A Powerful Technology Tool for the 21st Century Classroom". Theory into Practice, 220-228. $<$ http://digitalstorytellingclass.pbworks.com/f/Digital+Storytelling $+\mathrm{A}+$ Powerful.pdf $>$ [Consulta: 18 de marzo de 2016].

Robin, B. R. (2006). "The educational uses of digital storytelling”. Society for Information Technology \& Teacher Education International Conference. Vol. 2006, 1. $<$ https://digitalliteracyintheclassroom.pbworks.com/f/Educ-Uses-DS.pdf $>\quad[$ Consulta: $18 \mathrm{de}$ marzo de 2016].

SEvilla PAVÓN, A. (2015). "Examining collective authorship in collaborative writing tasks through digital storytelling". European Journal of Open and Distance Learning, 1: 1-6. $<$ http://www.eurodl.org/?p=current\&sp=brief\&article=685 $>$ [Consulta: 18 de marzo de 2016].

Sevilla Pavón, A., Serra Cámara, B. y Gimeno SAnz, A. (2012). "The Use of Digital Storytelling for ESP in a Technical English Course for Aerospace Engineers”. The Eurocall Review, 20 (2), 68$80 . \quad$ Disponible en:

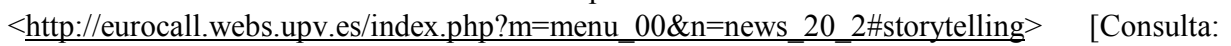
18 de marzo de 2016].

SmedA, N., DAKich, E. y ShaRdA, N. (2014). "The effectiveness of digital storytelling in the classrooms: a comprehensive study". Smart Learning Environments, 1 (6), 1-20.

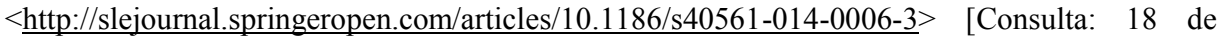
marzo de 2016].

VygOtSky, L. (1978). Mind and Society. Cambridge, Harvard University Press. 